\title{
COLONOSCOPY: A TWO YEAR EXPERIENCE FROM WESTERN NEPAL
}

\author{
Chaudhary S. ${ }^{1}$, Chaudhary P. ${ }^{2}$, Jaiswal N. ${ }^{1}$, Chaurasia R.K. ${ }^{3}$
}

\section{ABSTRACT}

INTRODUCTION: Colonoscopy remains the gold standard in diagnosing lower gastrointestinal pathologies. Colonoscopy is also helpful in preventing colorectal carcinoma by removing adenomatous polyp, however, colonoscopy is a difficult skill to master. Though Upper GI Endoscopy is widely available, colonoscopy facilities are relatively rare in developing world. In this field, the vast majority of reported data come from developed countries, and data from a developing country like Nepal is relatively scarce. With this background, present study was aimed to see clinical profile of patients presented for colonoscopy at a colonoscopy centre from western Nepal.

MATERIAL AND METHODS: It was a cross sectional study which was carried out in Endoscopy Centre, Edmond City Hospital, Butwal, Nepal from 1 October 2010 to 31 September 2012. Study was approved by the hospital authorities. All the patients for colonoscopy at our centre were evaluated as per predesigned Pro-forma. Colonoscopy was done in all the patients under intravenous sedation after bowel preparation. Colonoscopic abnormalities were noted and biopsied if indicated and sent for histopathological examination.

RESULTS: Sixty patients underwent colonoscopy in our centre during study period, among them 33 (55\%) were male and 27 (45\%) were female. The mean age was $45.23 \pm 15.30$ years (range 1678 years). Procedure was completed in $54(90 \%)$ patients. Altered bowel habit, chronic diarrhoea and bloody diarrhoea were the three most common indications for colonoscopy. Significant lesions were seen in 35 (56.67\%) patients. Among the patients with significant lesions anal canal or rectum or sigmoid colon were involved in $34(97.14 \%)$ patients. The most common finding in colonoscopy was nonspecific colitis involving rectum and sigmoid, seen in $8(13.33 \%)$ cases. Other findings include ulcerative colitis $6(10 \%)$, anal fissure $6(10 \%)$, haemorrhoids $5(8.33 \%)$, ca colon $4(6.67 \%)$, colonic polyp $4(6.67 \%)$, ca rectum $1(1.67 \%)$ and solitary rectal ulcer $1(1.67 \%)$ patients.

CONCLUSION: In present study altered bowel habit, chronic diarrhoea and bloody diarrhoea were the three most common indications for colonoscopy. Anal canal, rectum, sigmoid colon involvement were seen $97.14 \%$ patients. There is an urgent need to strengthen Lower GI Endoscopy facilities in resource poor setting. Flexible sigmoidoscopy may be a cheaper and easier initial screening tool for evaluating colorectal diseases.

KEY WORDS: Colonoscopy, Lower gastrointestinal endoscopy, Ulcerative colitis, Colon carcinoma, CRC Screening, Ulcerative Colitis.

1 Assistant Professor, Gastroenterology Division, Department of Internal Medicine, Universal College of Medical Sciences \& Teaching Hospital, Bhairahawa, Nepal

2 Pathologist, Lumbini Nursing Home, Butwal, Nepal

3 Department of Nephrology, National Academy of Medical Sciences, Bir Hospital, Kathmandu, Nepal

\author{
For Correspondence \\ Dr. Shatdal Chaudhary, M.D., \\ Assistant Professor, \\ Gastroenterology Division, Department of Internal Medicine, \\ Universal College of Medical Sciences \& Teaching Hospital, \\ Bhairahawa, Nepal \\ E-mail: shatdalchaudhary@yahoo.com
}




\section{INTRODUCTION}

Colonoscopy remains the gold standard in diagnosing lower gastrointestinal pathologies. Colonoscopy is a procedure used to see inside the colon and rectum and used in screening, assessment and management of colorectal diseases. Colonoscopy can detect inflamed tissue, ulcers, and abnormal growths. The procedure is used to look for early signs of colorectal cancer and help to diagnose unexplained changes in bowel habits, abdominal pain, bleeding from the anus, and weight loss. Colonoscopy has become popular after sixties of last century due to its increasing availability, relative safety and low complication rate. Colonoscopy is being commonly performed, incidence and prevalence of colonic diseases has been calculated in different geographical regions of the world. ${ }^{2}$ Among the various diagnostic tools available, colonoscopy is more sensitive than radiologic imaging and can be used for biopsy \& total resection of lesion. Colonoscopy is also helpful in preventing colorectal carcinoma by removing adenomatous polyp, however, colonoscopy is a difficult skill to master. The procedure is often painful for the patient. Over sedation, perforation, bleeding, and procedure related death remain much feared complications. ${ }^{3}$ It is widely used for diagnosis of colorectal cancers and adenomatous polyps that can be cancerous if they won't be managed. In this field, the vast majority of reported data come from North America, and data from developing country like Nepal is relatively scarce. Upper GI endoscopy facilities are easily available and regularly performed, but facilities for doing colonoscopy are very few and much less frequently performed in a resource poor country like Nepal. With this background, present study was aimed to see clinical profile of patients presented for colonoscopy at a colonoscopy centre from western Nepal.

\section{MATERIAL AND METHODS}

It was a cross-sectional study which was carried out in Endoscopy Centre, Edmond City Hospital, Butwal, Nepal from 1 October 2010 to 31 September 2012. Study was approved by the hospital authorities. All the patients for colonoscopy at our centre were evaluated. Written informed consent was taken. Detailed clinical history and physical examination including meticulous systemic examination was carried out in all the patients. Data was collected according to predesigned Pro-forma. Patients' data including age, sex, address, presenting symptoms, past history and treatment history was taken. Colonoscopy was done in all the patients. A liquid diet was recommended to patients 24 hours before the procedure. Bowel was prepared by 2-4 L of Polyethylene Glycol (PEG) solution. Patient was asked to drink PEG solution $12-18 \mathrm{hrs}$ before the examination after that patients were encouraged to drink electrolyte solution as much as possible. During procedure, patients were lied on their left side on an examination table and IV line was opened. Conscious sedation with 2.5-5 mg of intravenous Midazolam was used during procedure for all patients. Fuginon video colonoscope was used following standard high-level liquid disinfection with Cidex 2.5\% (Johnson \& Johnson, USA). Colonoscopic abnormalities were noted and biopsied if indicated and sent for histopathological examination. Heart-rate and oxygen saturation were monitored continuously by pulse oxymeter during the procedure. The data were entered in Microsoft Excel spreadsheet. The data was analyzed using SPSS version 11.5.0.

\section{RESULTS}

During the study period, 60 patients underwent colonoscopy in our centre. Among them 33 (55\%) were male and 27 (45\%) were female. The mean age was $45.23 \pm 15.30$ years (range 1678 years). Most of the patients were from surrounding districts Rupendehi 31 (51.66\%), Kapilvastu 11 (18.33\%), Palpa 7 (11.67\%), Nawalparasi 3 (5\%), Arghakhachi 3 (5\%), Gulmi 2 (3.33\%), Baglung 1 (1.67\%), Rolpa 1 (1.67\%) and Maharajgunj 1 (1.67\%). Forty patients $(66.67 \%)$ were referred by physicians while 19 patients $(31.67 \%)$ were referred by surgeons. One patient came himself for colonoscopy due to altered bowel habit. Mean duration of symptoms was $11.47 \pm$ 2.95 months (15 days to 10 years). Indication for doing colonoscopy is listed in table 1 .

\section{Table 1: Indications for colonoscopy}

\begin{tabular}{|l|l|}
\hline Indications for colonoscopy & Frequency (\%) \\
\hline Altered bowel habit & $22(36.67 \%)$ \\
\hline Chronic diarrhoea & $16(26.67 \%)$ \\
\hline Bloody diarrhoea & $07(11.67 \%)$ \\
\hline Bleeding PR & $05(8.33 \%)$ \\
\hline Constipation & $02(3.33 \%)$ \\
\hline Chronic abdominal pain & $03(5 \%)$ \\
\hline Follow up of ulcerative colitis & $01(1.67 \%)$ \\
\hline Follow up of Ca Colon & $02(3.33 \%)$ \\
\hline Follow up of post polypectomy & $01(1.67 \%)$ \\
\hline Abnormality on USG study & $01(1.67 \%)$ \\
\hline
\end{tabular}

Altered bowel habit and chronic diarrhoea and bloody diarrhoea were the three most common indications for colonoscopy. Procedure was completed in $54(90 \%)$ patients which was defined by the ability to visualize the caecum or terminal ileum. In $2(3.33 \%)$ patients colonoscope could not be passed after sigmoid due to malignant stricture and in another 
$4(6.67 \%)$ patients colonoscope could not be passed beyond splenic flexure. Significant lesions were seen in 35 (56.67\%) patients. The most common finding in colonoscopy was nonspecific colitis involving rectum and sigmoid, seen in 8 $(13.33 \%)$ cases. Other findings include ulcerative colitis 6 $(10 \%)$, anal fissure $6(10 \%)$, haemorrhoid $5(8.33 \%)$, ca colon $5(8.33 \%)$, colonic polyp $4(6.67 \%)$ and solitary rectal ulcer 1 (1.67\%) patients. (Figure: 1 )

Figure 1: Colonoscopy findings

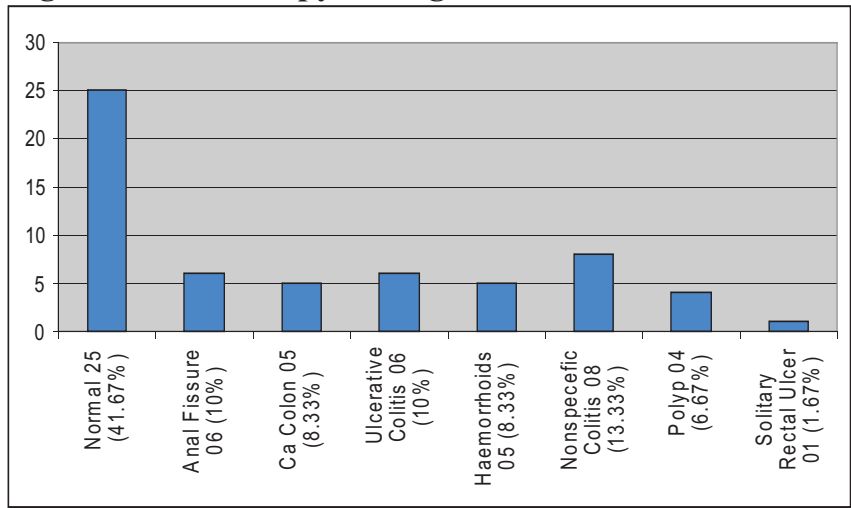

Among the patients with significant lesions, anal canal or rectum or sigmoid colon were involved in 34 (97.14\%) patients. There was only one patient where isolated involvement of colon proximal to sigmoid was seen in form of ca caecum. There were 6 cases of ulcerative colitis which were confirmed by histopathology. Among them in two patients disease was involving rectum and sigmoid, in one patient up to descending colon and in three patients there was pancolitis.

\section{DISCUSSION}

Colonoscopy is a procedure advocated in all the guidelines and advised for screening in patient more than 50 years of age. It is used for detecting colon's neoplasms like Familial Adenomatous Polyposis, Hereditary non-polypoid colon cancer and occult or gross blood in the stool in under 50. Other conditions where colonoscopy is indicated are GI bleeding, abdominal pain, anaemia, radiological abnormality, evaluation and follow up of inflammatory bowel disease, differentiating diverticular disease and malignancy, follow up of patient with prior colon surgery, confirmation of lesion location at the time of laparotomy and during laparoscopic procedures. ${ }^{4}$ In a national wide survey from France, the main indications for colonoscopy were asymptomatic colorectal cancer screening, history of colorectal polyp or cancer, positive faecal occult blood test, in 1619 (49.6\%) patients, abdominal symptoms (either change in bowel habit, constipation, diarrhoea, or abdominal pain) in 775 (23.7\%) patients, rectal bleeding in $496(15.2 \%)$ patients, and inflammatory bowel disease (at initial work-up or follow-up) in $86(2.6 \%)$ patients. ${ }^{5}$ Similarly in a retrospective study from eastern Nepal the most common indication for colonoscopy was irritable bowel syndrome to rule out organic disease (27.6\%). The other common indications were bleeding $\mathrm{P} / \mathrm{R}$ $(22.8 \%)$, chronic abdominal pain $(11.02 \%)$ and chronic diarrhoea $(11.02 \%){ }^{6}$

It is very important to achieve a good intestinal preparation, for good colonoscopic outcome. Insufficient colonoscopy preparation may lead to missed lesions, procedure failure, prolonged procedure time, and increased procedure complications. In an ideal intestinal preparation, the colon mucosa should be clearly seen in the colonoscopy, and there should not be any solid and liquid surplus. Colonoscopy preparation procedure should not cause visual or histological mucosal changes. The regimens should be acceptable by the patient. It should be effective, inexpensive and should not lead to fluid and electrolyte balance. ${ }^{7}$ Although the colonoscopy preparation regimens used currently are reliable, they sometimes does not meet the expectations. ${ }^{8}$ Sodium phosphate $(\mathrm{NaP})$ and $\mathrm{PEG}$ regimen are most commonly used. It has been demonstrated in some studies that polyethylene glycol is a faster, more effective and better-tolerated method for cleansing the colon. the Association of American Colon and Rectal Surgery (ASCRS), American Gastrointestinal Endoscopy Association (ASGE) and American Gastrointestinal and Endoscopic Surgery Association (SAGES) stated that $\mathrm{NaP}$ is an equal alternative with PEG except in those with heart failure, renal failure, hepatic failure, structural intestinal disorders, and in paediatric or elderly individuals. Most of the colonoscopists prefer NaP solutions over PEG or other solutions because it is effective in $30-45 \mathrm{ml}$ doses and its patient tolerability is higher. ${ }^{9}$ In a study from Ankara it was found that oral NaP solution for the purpose of preparation for colonoscopy was effective and well tolerated, with a low frequency of side effects in a selected group of patients (ambulatory patients without co morbidities such as heart failure, diabetes mellitus, chronic renal failure, or hepatic cirrhosis) aged over 70 years. ${ }^{10}$

Moderate sedation, using midazolam and an opioid, is the standard method of sedation, although propofol is increasingly being used in many countries because the satisfaction of endoscopists with propofol sedation is greater compared with their satisfaction with conventional sedation. Moreover, the use of propofol is currently preferred for the endoscopic sedation of patients with advanced liver disease due to its short biologic half-life and, consequently, its low risk of inducing hepatic encephalopathy. In the future, propofol could become the preferred sedation agent, especially for routine colonoscopy. Midazolam is the benzodiazepine of choice because of its shorter duration of action and better pharmacokinetic profile compared with diazepam. Among opioids, pethidine and fentanyl are the most popular. ${ }^{11}$ Sedation and analgesia is also associated with a small but definite risk of cardio-respiratory complications, which have been reported in $0.10 \%$ to $0.54 \%$ of patients, with fatalities 
occurring in $0.03 \%$. It is also associated with potential loss from work for the patient and the accompanying person and ultimately adds to the overall cost. Thus, the ability to perform colonoscopy without sedation and without compromising its efficacy rate could be highly advantageous in a developing country like Nepal. In a study from eastern Nepal colonoscopy without sedation was tolerated well and overall completeness rate of colonoscopy was $80.3 \% .^{12}$ In our study caecum was not reached in $6(10 \%)$ patients. This was similar to study from France where in $10.3 \%(336 / 3266)$ of cases, the colonoscopy did not reach the caecum.

Colorectal cancer (CRC) is the third most common cancer in both men and women worldwide and is the second most common cancer in Europe. Each year, more than 400,000 persons are diagnosed with $\mathrm{CRC}$ and more than half of them will die from the disease. ${ }^{13}$ The clinical and pathological stage at the time of diagnosis largely determines the prognosis of diagnosed patients. The CRC mortality rate could be decreased by the early detection of cancer, whereas both the mortality rate and the incidence can be decreased by the timely detection and removal of adenomatous polyps, precursor lesions of CRC. ${ }^{14}$

Due to poor colonoscopy facilities diagnosis of inflammatory bowel disease are often delayed and difficult. We diagnosed 6 biopsy proven cases of ulcerative colitis. Colonoscopy is used to make an initial diagnosis of inflammatory disease, distinguish crohn's disease from ulcerative colitis, assess the disease extent and activity, monitor response to therapy, allow for surveillance of dysplasia or neoplasia, and provide endoscopic treatment, such as stricture dilation. Individuals with long-standing UC and extensive CD colitis are at increased risk for development of dysplasia and colorectal cancer and should undergo colonoscopic surveillance. The risk of $\mathrm{CRC}$ increases with longer duration and extensive severe colitis, family history of CRC, young age at onset of disease, presence of backwash ileitis, and personal history of primary sclerosing cholangitis. Patients with UC or extensive Crohn's colitis (greater than one third colonic involvement) should undergo surveillance colonoscopy every 1 to 2 years beginning 8 to 10 years after disease onset. ${ }^{15}$ Biopsy specimens of the colon in patients with documented pancolitis should be obtained in all 4 quadrants every $10 \mathrm{~cm}$ from the cecum to the rectum, to obtain a minimum of 32 biopsy samples. ${ }^{15}$ Although colonoscopy of the terminal ileum is sufficient to differentiate the crohn's diseases and ulcerative colitis in most cases, approximately $10 \%$ of patients fall into the category of indeterminate colitis, which is relatively difficult to distinguish. When inflammation is severe, they become more difficult to distinguish. Crohn's disease is likely to be diagnosed among patients who manifest skip lesions, perianal lesions, and a cobblestone appearance, whereas a continuous granularity of the mucosa accompanied by erosions or diminutive ulcers is found more often with ulcerative colitis. ${ }^{16}$
We had one case of solitary rectal ulcer. Patient was $18 \mathrm{yr}$ old female presented with chronic mucoid diarrhoea of 12 month duration. There is a small retrospective case series from India where 22 children (age 18 months 18 years) diagnosed as solitary rectal ulcer syndrome were analysed. The majority $(81.8 \%)$ were $\geq 8$ years of age. Rectal bleeding was the presenting feature in all the children. Mucorrhea, constipation, tenesmus and rectal prolapse were observed in $77.3 \%, 63.6 \%$, $59 \%$ and $13.6 \%$ children, respectively. Colonoscopy showed classical single rectal ulcer in $68.2 \%$ and multiple ulcers in $22.7 \%$. Polypoidal and erosive lesions were documented in $4.5 \%$ each. $^{17}$

Colonoscopy is an invasive and burdensome procedure. There are other ways for diagnosing colorectal lesion. Double contrast barium enema (DCBE) was the first radiological exam that could evaluate the entire colon. DCBE coats the mucosal surface with high-density barium. CT colonography (CTC), also called virtual colonoscopy, allows an examination of the entire colon. Interpretation is made possible in twodimensional and three-dimensional images. CTC is considered a less invasive colonic exam compared with colonoscopy. If polyps or colorectal cancer are detected on CTC, a colonoscopy will follow for confirmation and, if possible, subsequent therapy. CTC screening leads to exposure of ionising radiation to asymptomatic persons. A low-dose protocol is regularly used and inherent chances of radiation-induced malignancy are low. Similarly Magnetic resonance imaging (MRI) of the colon has been increasingly studied in the last few years. This imaging technique also allows examination of the entire colon. The lack of ionising radiation and high soft tissue contrast could favour MRI over CTC. Accuracy of MR colonography in detecting colorectal polyps was evaluated in both high-risk and normal-risk cohorts. In a meta-analysis, its sensitivity in the detection of colorectal cancer was estimated at $100 \%$. For polyps with a size $\geq 10 \mathrm{~mm}$, per-patient sensitivity and specificity estimates were 88 and $99 \% .{ }^{18}$ Colon capsule endoscopy is a new technique to visualize the colon, originating from small bowel imaging. Colon capsule is an ingestible capsule consisting of an endoscope equipped with a video camera at both ends. Van Gossum et al. were the first to evaluate the effectiveness in a prospective setting. In high-risk patients, the sensitivity and specificity in detecting polyps $\geq 6 \mathrm{~mm}$ was 64 and $84 \%$ respectively and in detecting advanced adenomas 73 and $79 \%{ }^{19}$

\section{CONCLUSION}

In present study, we have shown clinical profile of patients presented for colonoscopy. Altered bowel habit, chronic diarrhoea and bloody diarrhoea were the three most common indications for colonoscopy. Anal canal, rectum, sigmoid colon involvement were seen $97.14 \%$ patients. The most common finding in colonoscopy was nonspecific colitis 
involving rectum and sigmoid, seen in $8(13.33 \%)$ cases other lesions found were ulcerative colitis $6(10 \%)$, anal fissure 6 (10\%), haemorrhoids $5(8.33 \%)$, ca colon $5(8.33 \%)$, colonic polyp $4(6.67 \%)$ patients. There is urgent need to strengthen Lower GI Endoscopy facilities in resource poor setting. So in resource poor setting sigmoidoscopy can be a cheaper and easier initial screening tool for evaluating colorectal diseases.

\section{ACKNOWLEDGEMENT}

We would like to thank Endoscopy and colonoscopy staff of Edmond City Hospital, Butwal, Nepal who assisted us in this study.

\section{CONFLICT OF INTEREST}

The authors declare that they have no conflict of Interest.

\section{REFERENCES}

1. Amjad S, Ammara A, Sobia Z, Arooj E. Colonoscopy: Analysis of Indications and Diagnoses at a Specialist Unit. Ann Pak Inst Med Sci 2010; 6: 15-19.'

2. Wong RF, Khosla R, Moore JH, Kuwada SK. Consider colonoscopy for young patients with hematochezia. The Journal of Family Practice 2004; 53: 879-884.

3. Nahas SC, Alves PR, Araujo SE, Silva e Souza, Júnior AH, da Sobrado Júnior CW, Nahas CS, Haber-Gama A, Pinotti HW. Colonoscopy as a diagnostic and therapeutic method in large intestine diseases. Results of 1715 exams. Rev Hosp Clin Fac Med Sao Paulo 1998; 53: 117-121.

4. Huang EH, Marks JM. The diagnostic and therapeutic roles of colonoscopy. Surg Endosc 2001; 15: 1373-1380.

5. Barret M, Boustiere C, Canard J, Arpurt J, Bernardini D et al. (2013) Factors Associated with Adenoma Detection Rate and Diagnosis of Polyps and Colorectal Cancer during Colonoscopy in France: Results of a Prospective, Nationwide Survey. PLoS ONE 8(7): e68947. doi:10.1371/journal.

6. Pradhan B, Pandey N. Colonoscopy in patients without sedation. Health Renaissance, January-April 2012; Vol 10 (No. 1);27-292.

7. Tooson JD, Gates LK Jr. Bowel preparation before colonoscopy. Choosing the best lavage regimen. Postgrad Med 1996; 100: 203-4, 207-12, 214.
8. Linchtensstein G. Bowel preparations for colonoscopy: a review. Am J Health Syst Pharm 2009; 66: 27-37.

9. Steven DW, David EB, Todd HB et al. A consensus document on bowel preparation before colonoscopy: prepared by a task force from the American Society of Colon and Rectal Surgeons (ASCRS), the American Society for Gastrointestinal Endoscopy $(A S G E)$, and the Society of American Gastrointestinal and Endoscopic Surgeons (SAGES). Surg Endosc 2006; 20: 1147-60.

10. Yakut $M$, Çinar K, Seven $G$, Çetinkaya H, KaBahar K. Turk J Gastroenterol 2010; 21 (2): 140-145.

11. Triantafillidis JK, Merikas E, Nikolakis D, Papalois AE. Sedation in gastrointestinal endoscopy: Current issues. World J Gastroenterol2013; 19(4): 463-481.

12. Arrowsmith JB, Gerstman BB, Fleischer DE, Benjamin SB. Results from the American Society for gastrointestinal Endoscopy/ US. Food and Drug Administration collaborative study on complication rates and drug use during gastrointestinal endoscopy. Gastrointest Endosc 1991; 37: 421-7.

13. Ferlay J, Autier P, Boniol M, Heanue M, Colombet M, Boyle P. Estimates of the cancer incidence and mortality in Europe in 2006. Ann Oncol. 2007 Mar;18(3):581-92.

14. Winawer SJ, Zauber AG, Ho MN, O'Brien MJ, Gottlieb LS, Sternberg SS et al. Prevention of colorectal cancer by colonoscopicpolypectomy. The National Polyp Study Workgroup. NEnglJMed. 1993 Dec 30;329(27):1977-81.

15. Itzkowitz SH, Present DH. Crohn's and Colitis Foundation of America Colon Cancer in IBD Study Group: consensus conference: colorectal cancer screening and surveillance in inflammatory bowel disease. Inflamm Bowel Dis 2005;11:314-21.

16. Kim YH. Common errors in the diagnosis process. Intest Res 2003;1: 106-110.

17. Suresh N, Ganesh R, Sathiyasekaran M. Solitary Rectal Ulcer Syndrome: A Case Series. Indian Pediatrics : Dec 2010;47:1059 1061.

18. Zijta FM, Bipat S, Stoker J. Magnetic resonance (MR) colonography in the detection of colorectal lesions: a systematic review of prospective studies. Eur Radiol. 2010 May;20(5):1031-46.

19. Van Gossum A, Munoz-Navas M, Fernandez-Urien I, Carretero $C$, Gay G,Delvaux $M$ et al. Capsule endoscopy versus colonoscopy for the detection of polyps and cancer. $N$ Engl $J$ Med. 2009 Jul 16;361(3):264-70. 